\title{
Contributions of Peer Support to Health, Health Care, and Prevention: Papers from Peers for Progress
}

\author{
Edwin B. Fisher, $P b D^{1,2}$ \\ Guadalupe X. Ayala, PbD, MPH \\ Leticia Ibarra, MPH \\ Andrea L. Cherrington, $M D, M P H^{5}$ \\ Jobn P. Elder, $\mathrm{PbD}, \mathrm{MPH}{ }^{3}$ \\ Tricia S. Tang, $P b D^{6}$ \\ Micbele Heisler, MD, MPA \\ Monika M. Safford, $M D^{5}$ \\ David Simmons, $M D^{8,9}$
}

For the Peers for Progress Investigator Group

'Peers for Progress, American Academy of Family Physicians Foundation, Leawood,

Kansas

${ }^{2}$ Department of Health Behavior, Gillings School of Global Public Health, University of North Carolina at Chapel Hill, Chapel Hill, North Carolina

${ }^{3}$ San Diego State University College of Health and Human Services and Institute for Behavioral and Community Health, San Diego State University Research Foundation, San Diego, California

${ }^{4}$ Clínicas de Salud del Pueblo, Inc., Brawley, California

${ }^{5}$ Department of Medicine, School of Medicine, University of Alabama at Birmingham, Birmingham, Alabama

${ }^{6}$ University of British Columbia Department of Medicine, Vancouver, British Columbia

${ }^{7}$ University of Michigan Department of Internal Medicine, Ann Arbor, Michigan ${ }^{8}$ School of Medicine, University of Western Sydney, Australia

${ }^{9}$ Institute of Metabolic Science, Cambridge University Hospitals, NHS Foundation

Trust, Cambridge, England

Conflicts of interest: Fisher receives salary support for bis role as Global Director of Peers for Progress from the American Academy of Family Physicians Foundation and also received compensation for serving as a consultant for the Foundation for activities unrelated to this paper.

\section{CORRESPONDING AUTHOR}

Edwin B. Fisher, $\mathrm{PhD}$

Department of Health Behavior

Gillings School of Global Public Health

University of North Carolina-Chapel Hill

Chapel Hill, NC 27599-7440

edfisher@unc.edu

\begin{abstract}
SUBSTANTIAL evidence documents the benefits of peer support provided by community health workers, lay health advisors, promotores de salud, and others. The papers in this supplement, all supported by the Peers for Progress program of the American Academy of Family Physicians Foundation, contribute to the growing body of literature addressing the efficacy, effectiveness, feasibility, reach, sustainability, and adoption of peer support for diabetes self-management. They and additional papers supported by Peers for Progress contribute to understanding how peer support can be implemented in real world settings. Topics include examination of the peers who provide peer support, reaching the hardly reached, success factors in peer support interventions, proactive approaches, attention to emotions, peer support in behavioral health, dissemination models and their application in China, peer support in the patient-centered medical home, research challenges, and policy implications.
\end{abstract}

Ann Fam Med 2015;13(Suppl_1):S2-S8. doi: 10.1370/afm.1852.

W hy peer support? Social support is a powerful force in human behavior $^{1}$ and health. ${ }^{2,3}$ Peer support can be provided by community health workers, lay health advisors, promotores de salud, and individuals with a number of other titles, for people with a variety of health needs. In addition to earlier papers, ${ }^{4,5}$ a 2014 review identifies contributions of community health workers to supplying basic health needs (eg, reducing childhood undernutrition), to primary care and health promotion, and to disease management. ${ }^{6}$ Emerging economic analyses of peer support also show diverse benefits. ${ }^{7-13}$ Accordingly, community health workers receive strong emphasis in the World Health Organization's Global Health Workforce Alliance 5 and are prominently mentioned in various provisions of the US Patient Protection and Affordable Care Act. ${ }^{14}$

The American Academy of Family Physicians Foundation, with support from the Eli Lilly and Company Foundation, initiated Peers for Progress (http://peersforprogress.org) in 2006 with goals of promoting peer support for those with diabetes and other health challenges around the world.${ }^{15} \mathrm{~A}$ program development center was established in 2008 at the Gillings School of Global Public Health at the University of North Carolina at Chapel Hill. Recognizing the need to build the evidence base for peer support and establish working program models, Peers for Progress awarded 14 grants in 9 countries on 6 continents in $2009 .{ }^{16}$ In 2010, a major award from the Bristol-Myers Squibb Foundation supported, among other related activities, a demonstration of the integration of peer support into the patient-centered medical home $(\mathrm{PCMH})$ in collaboration with the National Council of $\mathrm{La}$ Raza. Papers from these projects have documented the efficacy of peer support. ${ }^{17-21}$ The present supplement to the Annals of Family Medicine includes additional evaluations of peer support interventions as well as papers addressing a number of issues in extending the reach and benefit of peer support. The accomplishments of Peers for Progress and the value of these latest additions to them can be clearly seen against the backdrop of the evidence needed to drive practical applications of peer support. ${ }^{22,23}$ 


\section{A PRACTICAL, FUNCTIONAL DEFINITION FOR DISSEMINATION}

Chronic disease care in general and peer support in particular are so strongly shaped by culture, setting, and population that the dissemination of peer support inevitably involves a tension between standardization and flexibility. Standardization of essential or defining features is important. On the other hand, flexibility is required to accommodate cultural and population differences and varying systems of care. ${ }^{24}$

Representatives of over 20 countries, organized through the World Health Organization in 2007, noted that although individual programs need to be tailored to the systems, cultures, and populations in which they are applied, standard features of peer support can be identified.$^{25}$ Accordingly, Peers for Progress has pursued a strategy of defining peer support not by who provides it nor by specific implementation protocols but according to 4 "key functions of support." 24 This follows a strategy of "standardization by function, not content." 26 The 4 key functions are these:

- Assistance in daily management such as in working out specific plans for pursuing goals developed with clinical team

- Social and emotional support to encourage management behaviors and coping with negative emotions

- Linkage to clinical care and community resources

- Ongoing support reflecting the lifelong nature of prevention and chronic disease management ${ }^{16}$

The 4 key functions serve as a template for planning and evaluating peer support programs. ${ }^{27}$

\section{FEASIBILITY, REACH, SUSTAINABILITY AND ADOPTION OF PEER SUPPORT}

\section{Feasibility}

Of the 14 Peers for Progress projects that involved implementation of peer support programs, 9 provided care in under-resourced settings or health systems (4 in the United States, 2 in Cameroon, and 1 each in South Africa, Uganda, and Thailand), with the remaining 5 representing Argentina, Australia, Hong Kong, the United Kingdom, and an insured population in the United States. This range of settings speaks to the broad feasibility of peer support in varied cultures and populations.

Across those 14 projects, the number of participants receiving peer support ranged from 46 to 810 , and the average retention of participants for follow up evaluations was $78.6 \%$ (range $45.7 \%$ to $99.4 \%$ ). The average initial hemoglobin $\mathrm{A}_{\mathrm{cc}}\left(\mathrm{HbA}_{\mathrm{lc}}\right)$ was $8.41 \%$. Given typical targets of $<6.5$ or $<7.0 \%,{ }^{28}$ the programs appear to have reached those for whom improved diabetes management is important.
Individual studies illustrate these broader trends. In Cambridgeshire, UK, 1,299 people enrolled for the peer support program, and 167 of these trained as peer support facilitators. ${ }^{20}$ In a 6 -month program in Cameroon, only 1 of 100 participants dropped out. ${ }^{27}$ Emphasizing community partnerships, a peer support program for low-income African Americans in underserved, rural Alabama communities exceeded its recruitment goal of 400 participants. ${ }^{29}$

Remarkably, studies have shown peer support to be most successful in "hardly reached" groups amongwhom one might expect the least success. ${ }^{30}$ In a peer support intervention for diabetes management among ethnic minority patients of safety-net clinics in San Francisco, 17 participants were categorized at baseline as low, medium, or high on medication adherence and on self management. The greatest differential benefit of peer support relative to usual care control was among those categorized as low on medication adherence or self management. ${ }^{31}$ In a peer support intervention for diabetes management among ethnic minority patients of safety-net clinics in San Francisco, ${ }^{17}$ participants were categorized at baseline as low, medium or high on medication adherence and on self management.

\section{Efficacy and Effectiveness}

As noted above, a number of papers from the Peers for Progress investigators have documented benefits of peer support. ${ }^{17-21}$ Three papers in this supplement add to this evidence. Ayala and colleagues, ${ }^{32}$ evaluated peer support provided by trained volunteers for Latino adults with diabetes in Southern California near the border with Mexico. Although supporters were able to make an average of only 4 contacts per participant out of the 8 anticipated in the protocol, significant reductions in $\mathrm{HbA}_{1 \mathrm{c}}$ were observed relative to those in usual care. The paper by Safford and colleagues shows significant stabilization of cardiovascular risk factors over time for participants receiving peer support compared to controls among African American residents of rural Alabama, an area of great poverty and poorly organized clinical care. ${ }^{33}$ Tang and her colleagues have focused on peer support as a way of helping to sustain gains from diabetes self-management education. In an earlier paper concerning peer support for Latinos with diabetes, they showed that both staff community health workers and trained volunteer peer supporters were successful in this. ${ }^{19}$ Their paper in this supplement shows that peer-led group sessions and additional telephone support from peer-led groups for African Americans in urban Michigan were successful in improving low density lipoprotein levels, systolic and diastolic blood pressure, and body mass indexalthough, surprisingly, not $\mathrm{HbA}_{1 \mathrm{c}}{ }^{34}$ 


\section{Program Sustainability and Spread}

A number of the 14 projects funded by Peers for Progress have been sustained and have extended their work beyond the original study groups. Four years after the end of funding from Peers for Progress, local provincial administrative offices in Thailand committed cash and in-kind support to ensure the sustainability of village health volunteers providing diabetes education and support for which they had been trained. The administrative offices noted the values of the project not only in terms of health, but also in building unity and sense of belonging among people. In light of successes with diabetes described below, the Anhui Provincial Health Bureau in China has extended a peer support program to additional communities and to cardiovascular disease prevention and management. ${ }^{35}$

New funding from the UK government and collaboration with Diabetes UK will extend the program from Cambridgeshire ${ }^{20}$ to 8 areas in the Eastern region and West Midlands. After its own initial appraisal of 15 sites, Texas-based WellMed extended its peer support program to all 23 of the clinical sites in its network. ${ }^{36}$ In Clinicas de Salud del Pueblo in southern California, the Puentes bacia una major vida program for adults with diabetes was used as a model for addressing childhood obesity and helped to guide changes to the clinics' system of care, including provider training and EHR changes to ensure documentation of peer support. ${ }^{32}$

\section{SCALING UP}

If peer support programs are feasible, reach and engage important groups, achieve benefits, and can be adopted and sustained by existing organizations, the next step is to understand better how peer support works and how it can be scaled up for implementation in realworld settings. Key findings curated by Peers for Progress and reported by papers in this supplement ${ }^{35-41}$ help to guide these efforts.

\section{Looking at the Peer Supporters Themselves}

Two questions frequently raised concerning peer support programs are whether, with brief training, lay people can pick up the skills and knowledge necessary to provide effective support and whether providing peer support has beneficial effects on the peer supporters themselves. Previous research by Tang and her colleagues working through African American churches showed the feasibility of training nonprofessionals to provide peer support for both diabetes prevention and management. ${ }^{42}$ Two papers in this supplement explore these issues further. Goldman and colleagues examined the degree to which peer supporters actually used ask-tell-ask strategies, action planning, and other components of an evidence-based health coaching curriculum. ${ }^{37}$ Although the coaching intervention was successful in reducing $\mathrm{HbA}_{1 \mathrm{c}}{ }^{17}$ and the coaches wanted to continue their work and expressed confidence in their abilities, they did not consistently use the coaching techniques they had been taught.

As part of a project led by Chan in Hong Kong, ${ }^{21}$ Yin and colleagues studied the effects of providing peer support on the $\mathrm{HbA}_{1 \mathrm{c}}$ levels of a group of peer supporters. Their changes over time were compared with those of a group of individuals who were trained but then declined to work as peer supporters and those of a group of patients with comparable glycemic control who did not attend the training program. Although diabetes is a progressive disease, the researchers found that the $\mathrm{HbA}_{1 \mathrm{c}}$ values of those who served as peer supporters increased only slightly, from $7.0 \%$ to $7.2 \%$ over 4 years. This contrasted sharply with increases from $7.1 \%$ to $7.8 \%$ in those who were trained but declined to work as peers and from $7.1 \%$ to $8.1 \%$ among the comparison patients who did not attend training. ${ }^{38}$

\section{The 4 Key Functions in China}

Dissemination of peer support programs in China needs to respect preferences that programs be "made in China"; that is, that Chinese collaborators lead actual planning and development. Emphasizing the 4 key functions rather than a concrete program plan has facilitated a number of such collaborations number of collaborationIs such as with the Chinese Diabetes Society, Zhongda Hospital and Southeast University in Nanjing, the Beijing Diabetes Prevention \& Treatment Association, and the Shanghai Sixth People's Hospital.

A demonstration project in community health centers in Anhui province, described by Zhong and colleagues in this supplement, set a model for adaptation of peer support to Chinese needs and culture. ${ }^{35}$ The program trained mostly retired adults with diabetes to co-lead monthly educational meetings with staff of community health centers. The peer leaders also led discussion groups that provided more opportunity for participants to talk about self-management plans, obstacles encountered, and successes. The peer leaders also promoted informal groups within housing complexes such as for tai chi, morning walking, shopping, and even fishing. Reported advantages included the way peer support provided a "bridge" between community health centers and their patients. The program achieved significant benefits relative to controls $(P<.05)$ for knowledge, self efficacy, body mass index, systolic and diastolic blood pressure, and both fasting and 2-hour post-prandial blood glucose among partici- 
pants who had completed both baseline and follow-up assessments. ${ }^{35}$

\section{Peer Support and the Patient-Centered Medical Home}

A key objective of the PCMH is to engage the whole person in the context of the values, interests, family, and community that frame individual lives. Substantial evidence shows that peer support can help in this. The present paper by Daaleman and Fisher ${ }^{39}$ seeks to "accelerate the integration" of peer support into PCMHs by addressing 3 elements: a model of peer support that stresses the 4 key functions identified by Peers for Progress, a framework and strategies for implementation, and fiscal models to sustain these approaches.

Much research on peer support has focused on its value for reaching disadvantaged groups and reducing disparities. The present paper by Knox and her colleagues raises the question of the added value of peer support for those already receiving good patient care. The Carpeta Roja peer mentoring program, developed for mostly underserved groups in Los Angeles, was modified for the older adult, ensured Latinos in the intended audience in Texas. All participants were offered diabetes education, but those who participated in peer mentoring as well showed more rapid improvement in glycemic control and also reported reductions in social isolation and the extension of benefits to their families. ${ }^{36}$

Using the PCMH as a base for peer support was the focus of a collaboration of Peers for Progress with the National Council of La Raza, TransforMED, and Alivio Medical Center, a federally qualified health center serving predominantly Latinos in Chicago. The program, Compañeros en Salud, was closely coordinated with clinical teams, providing individualized as well as group patient education and support. Patients were identified through those clinical teams and medical records. Physicians also identified patients likely to benefit from individualized contacts and communicated regularly with Compañeros staff regarding individual patients and programmatic issues. The program also sought to develop a model for reaching the entire population of those with diabetes served by Alivio, in this case 3,787 adults. To do so, it distinguished 471 high need patients based on elevated $\mathrm{HbA}_{1 \mathrm{c}}$ levels, depression or psychosocial distress, or physician referral. These patients received individual peer support starting biweekly and then less frequently as progress warranted. The balance of those receiving regular care were offered group education and support as well as individual contacts such as in conjunction with clinic visits. All patients could also contact the Compañeros at their own initiative. Overall, the Compañeros reached over $80 \%$ of each of the high need and regular care groups, showing the utility of the $\mathrm{PCMH}$ as a base for staging peer support. Although modest, reflecting the inclusion of all with diabetes, not just a research sample, clinical impacts were significant, eg, declines of $\mathrm{HbA}_{1 \mathrm{c}}$ from $8.22 \%$ to $8.14 \%$ over 2 years across the entire population of those with diabetes, $P<.05$; from $9.43 \%$ to $9.16 \%$ in the high need group, $P=.01$.

\section{Research Challenges}

Research on peer support in real-world settings entails numerous challenges to conventional research designs. These include integrating complex, multi-faceted interventions (eg, group sessions, individual sessions, telehealth channels, variable schedules and numbers of contacts, topics tailored to individuals' needs and attention to emergent concerns of the individual) and linking with other resources in the community and clinical settings (eg, group medical visits). Clearly, evaluation of how peer support may be adapted to "real world" populations and settings cannot be easily fit into conventional clinical research designs.

One aspect of real-world diabetes that research rarely addresses is that it changes. Safford and Richman and their colleagues in Alabama have explored the importance of seasonality and changes over time. In addition to applying these perspectives in analyzing their intervention, noted above, ${ }^{33}$ Richman has led additional work to outline analytic approaches that accommodate the seasonal and nonlinear, dynamic characteristics of diabetes as well as research challenges common in real world settings such as variability of time between baseline and follow-up measures and secular trends. ${ }^{40}$

Peer support and other community-focused interventions, especially those intending to reach populations that more conventional approaches fail to engage, raise a number of ethical issues, including confidentiality and informed consent. Simmons led the development of a paper with input from a number of other investigators funded by Peers for Progress that examined ethical issues surrounding peer support and how committees for protection of human subjects review studies in this area. As a measure of the appropriateness of ethical review, the authors used a standard framework of ethical issues (the " $4+1$ Framework") that includes consideration of autonomy, beneficence, non-maleficence, and justice, as well as the scope of application. ${ }^{43}$ They identified 20 themes that emerged in the ethical reviews of 8 projects, but they found that only 4 of these fell within the $4+1$ Framework. Instead, a variety of other considerations were applied by ethics committees, including scientific, organizational and administrative issues that sometimes delayed studies or (of special concern for 
research seeking to reach and engage diverse groups) increased participant burden and mandated consent processes that could hinder the responsiveness of peer support to diverse needs-as the paper concludes, "creating a new ethical dilemma." ${ }^{\text {"1 }}$

\section{LOOKING FORWARD}

In addition to the findings detailed in this supplement, a number of other features of peer support have substantial implications for taking it to scale. ${ }^{44}$

\section{Connection to Community}

Long a major emphasis of community health workers ${ }_{1}{ }^{45}$ peer support may gain strength through the experiences those providing it share with communities. This also constitutes a base for linking communities, individuals, and health centers. ${ }^{46}$ As noted in the description of the program in Anhui, China above, one important contribution of peer leaders is the way they can serve as "the bridge to link [the clinic] with patients in their served neighborhood." ${ }^{\prime 35}$

\section{Emotional Support}

Anecdotally, many patients who consider participating in a peer support program express an interest in getting answers to practical questions, not discussing their feelings. However, interviews with peer supporters in projects in the $\mathrm{UK}^{20}{ }^{20} \mathrm{Chicago}^{44}$, and North Carolina ${ }^{47}$ showed that, in practice, emotional support evolves over time, emerging out of instrumental support and the trust it builds. ${ }^{48}$ An additional important feature of peer support is implicit emotional support, ${ }^{49}$ the expression of emotional reassurance, acceptance and similar messages not through explicit discussion of feelings but through sharing of activities, discussions of common interests and the like.

The universality of emotional support was suggested in the program in Anhui province ${ }^{35}$ in which a number of participants reported valuing the opportunity to share feelings with their peer supporters. In China and other Asian countries, cultural emphases on the fundamental commitment of the family to its members brings a reluctance to obligate family members with one's own concerns. ${ }^{49}$ Peer leaders, however, are not connected to participants as family members are and so provide an outlet for emotional expression free of concerns about "burdening" others.

\section{Behavioral Health}

Recognition of the role of emotional support in peer support also leads to consideration of the role of peer support in addressing the psychological distress and mental health problems that so often complicate chronic disease and impair quality of life. In a remarkable demonstration of potential benefit, a randomized study by Chan and her colleagues in Hong Kong evaluated structured care for diabetes alone ${ }^{50}$ and with peer support for diabetes management. Approximately $20 \%$ of the sample were above norms for depression, anxiety, and stress and also accounted for highly disproportionate rates of hospitalization. In this $20 \%$, peer support not only reduced distress substantially, but also lowered rates of hospitalization to the levels of individuals not similarly distressed. ${ }^{21}$ In addition to its implications for health systems' concerns about avoidable, costly care among those with multiple morbidities and psychological distress, it is remarkable that this peer support intervention was not designed to address emotional issues but to focus on diabetes management. Similar findings of emotional and quality-of-life benefits of other peer support interventions designed for diabetes management have been noted ${ }^{51}$ suggesting core characteristics of peer support that may be important in this area of concern for "bending the curve" of health care costs and benefits.

\section{CONCLUSIONS AND POLICY IMPLICATIONS}

A 2014 international meeting hosted by Peers for Progress and addressing much of this work ${ }^{52-54}$ noted that, in addition to its reach and effectiveness, peer support fills a critical role in humanizing health care. This dual role of enhancing effectiveness and humanizing care is an important feature of peer support for prevention and health care of the future.

Beyond showing that it works, emerging research shows that peer support is broadly feasible and sustainable. Dissemination can combine standardization, for instance through the 4 key functions emphasized here, and adaptability to local circumstances. Keys to its success are becoming clear, including both personand community-centered implementation, proactive offering of services, and attention to emotional as well as instrumental support. Most important, perhaps, peer support shows strong potential for addressing key priorities in "bending the curve" through health care reform: reaching those whom conventional services too often fail to engage, mitigating and reducing costs associated with concurrent physical and psychological problems, and providing intervention models adaptable to diverse settings and populations. ${ }^{55}$

To read or post commentaries in response to this article, see it online at http://www.annfammed.org/content/13/Suppl_1/S2.

Funding support: Funding for this research was provided by the American Academy of Family Physicians Foundation through the Peers for Progress program with support from the Eli Lilly and Company Foundation and the Bristol Myers Squibb and Company Foundation. 
Acknowledgments: This paper would not have been possible without the work of the investigators supported by grants from Peers for Progress. In alphabetical order according to organization, they are:

American Academy of Family Physicians National Research Network, LA Net, WellMed

Lyndee Knox, PhD; Wilson Pace, MD; America Bracho, MD, MPH

CENEXA - Centro de Endocrinologia Experimental y Aplicada, Universidad Nacional de La Plata (UNLP-CONICET), La Plata,

Argentina Juan José Gagliardino, MD; Charles M. Clark, Jr, MD

Centre for Population Studies and Health Promotion, University of Yaounde, Cameroon

Paschal Kum Awah, PhD; Andre-Pascal Kengne, MD, PhD

Hong Kong Institute of Diabetes and Obesity, The Chinese University of Hong Kong, Prince of Wales Hospital

Juliana C.N. Chan, MD; Rebecca Y.M. Wong, MSC; Gary T.C. Ko, MD; Roseanne O. Yeung, MD,MPH; Suky Junmei Yin, MPH

Institute of Metabolic Science, Cambridge University Hospitals, University of Cambridge, Cambridge, England

David Simmons, FRACP, FRCP, MD; Jonathan P. Graffy, FRCGP, MD

Mahidol University, Bangkok

Boosaba Sanguanprasit, PhD, MPH; Chanuantong Tanasugarn, DrPH $\mathrm{MPH}$

Monash University and DiabetesVic, Melbourne, Australia Brian Oldenburg, PhD; Michaela Riddell, PhD

\section{San Diego State University}

Guadalupe X. Ayala, PhD, MPH; Andrea Cherrington, MD, MPH; John P. Elder, PhD, MPH; Lucy Horton, MS, MPH; Leticia Ibarrra, MPH

School of Medicine, University of Alabama at Birmingham, Birmingham, Alabama

Monika M. Safford, MD; Andrea Cherrington, MD, MPH; Susan

Andreae, MPH

School of Nursing, University of Wisconsin-Madison, Madison, Wisconsin; Mulago Hospital, Kampala, Uganda

Linda C. Baumann, RN, PhD; Fred Nakwagala, MD

University of California, Los Angeles; Women for Peace, Western Cape, South Africa

Mary Jane Rotheram-Borus, PhD; Margaret Gwegwe, BA; Neal

Kaufman, MD, MPH

\section{University of California, San Francisco}

Thomas Bodenheimer, MD, MPH; David H. Thom, MD, PhD; Ellen Chen, MD; Amireh Ghorob, MPH; Danielle Hessler, PhD

\section{University of Michigan}

Michele Heisler, MD, MPA; Tricia S. Tang, PhD; Martha M. Funnell, MS, RN, CDE; Robin Nwankwo, RD, MPH, CDE

University of Yaounde and Central Hospital, Yaounde, Cameroon Jean Claude Mbanya, MD, PhD, FRCP; Felix K. Assah, MD, PhD

\section{REFERENCES}

1. Harlow HF, Harlow M. Learning to love. Am Sci. 1966;54(3):244-272.

2. Holt-Lunstad J, Smith TB, Layton JB. Social relationships and mortality risk: a meta-analytic review. PLoS Med. 2010;7(7):e1000316.

3. House JS, Landis KR, Umberson D. Social relationships and health. Science. 1988:241(4865):540-545.
4. Viswanathan M, Kraschnewski JL, Nishikawa B, et al. Outcomes and costs of community health worker interventions: a systematic review. Med Care. 2010;48(9):792-808.

5. Bhutta Z, Lassi Z, Pariyo G, Huicho L. Global Experience of Community Health Workers for Delivery of Health Related Millennium Development Goals: A Systematic Review, Country Case Studies, and Recommendations for Integration Into National Health Systems. Geneva: Global Health Workforce Alliance; 2010.

6. Perry HB, Zulliger R, Rogers MM. Community health workers in low-, middle-, and high-income countries: an overview of their history, recent evolution, and current effectiveness. Annu Rev Public Health. 2014;35:399-421.

7. Whitley EM, Everhart RM, Wright RA. Measuring return on investment of outreach by community health workers. J Health Care Poor Underserved. 2006;17(1)(Suppl):6-15.

8. Brownson CA, Hoerger TJ, Fisher EB, Kilpatrick KE. Costeffectiveness of diabetes self-management programs in community primary care settings. Diabetes Educ. 2009;35(5):761-769.

9. Margellos-Anast H, Gutierrez MA, Whitman S. Improving asthma management among African-American children via a community health worker model: findings from a Chicago-based pilot intervention. J Asthma. 2012;49(4):380-389.

10. Brown HS III, Wilson KJ, Pagán JA, et al. Cost-effectiveness analysis of a community health worker intervention for low-income Hispanic adults with diabetes. Prev Chronic Dis. 2012;9:E140.

11. Sledge WH, Lawless M, Sells D, Wieland M, O'Connell MJ, Davidson L. Effectiveness of peer support in reducing readmissions of persons with multiple psychiatric hospitalizations. Psychiatr Serv. 2011;62(5):541-544.

12. Patel V, Weiss HA, Chowdhary N, et al. Lay health worker led intervention for depressive and anxiety disorders in India: impact on clinical and disability outcomes over 12 months. Br J Psychiatry. 2011;199(6):459-466.

13. Peers for Progress. Economic Analysis in Peer Support: Breadth of Approaches and Implications for Peer Support Programs: American Academy of Family Physicians Foundation; 2014. http://peersforprogress.org/wp-content/uploads/2015/04/150417-economic-analysis-in-peer-support.pdf. Accessed August 5, 2015.

14. Peers for Progress. Opportunities for Peer Support in the Affordable Care Act [issue brief]. http://peersforprogress.org/wp-content/ uploads/2012/12/140728-peer-support-and-the-affordable-careact-2014.pdf. Updated June 2014. Accessed August 5, 2015.

15. Caro JF, Fisher EB. A solution might be within people with diabetes themselves. Fam Pract. 2010;27(Suppl 1):i1-i2.

16. Boothroyd RI, Fisher EB. Peers for progress: promoting peer support for health around the world. Fam Pract. 2010;27(Suppl 1):i62-i68.

17. Thom DH, Ghorob A, Hessler D, De Vore D, Chen E, Bodenheimer TA. Impact of peer health coaching on glycemic control in lowincome patients with diabetes: a randomized controlled trial. Ann Fam Med. 2013;11(2):137-144.

18. Gagliardino JJ, Arrechea V, Assad D, et al. Type 2 diabetes patients educated by other patients perform at least as well as patients trained by professionals. Diabetes Metab Res Rev. 2013;29(2):152-160.

19. Tang TS, Funnell $M$, Sinco $B$, et al. Comparative effectiveness of peer leaders and community health workers in diabetes selfmanagement support: results of a randomized controlled trial. Diabetes Care. 2014;37(6):1525-1534.

20. Simmons D, Prevost AT, Bunn C, et al. Impact of community based peer support in type 2 diabetes: a cluster randomised controlled trial of individual and/or group approaches. PLoS One. 2015;10(3): e0120277.

21. Chan JC, Sui Y, Oldenburg B, et al.; JADE and PEARL Project Team. Effects of telephone-based peer support in patients with type 2 diabetes mellitus receiving integrated care: a randomized clinical trial. JAMA Intern Med. 2014;174(6):972-981. 
22. Green LW, Kreuter MW. Health Promotion Planning: An Educational and Ecological Approach. 3rd ed. Mountain View, CA: Mayfield; 1999.

23. Glasgow RE, Nelson CC, Strycker LA, King DK. Using RE-AIM metrics to evaluate diabetes self-management support interventions. Am J Prev Med. 2006;30(1):67-73.

24. Fisher EB, Earp JA, Maman S, Zolotor A. Cross-cultural and international adaptation of peer support for diabetes management. Fam Pract. 2010;27(Suppl 1):i6-i16.

25. World Health Organization. Peer Support Programmes in Diabetes: Report of a WHO Consultation. Geneva: World Health Organization; 2008.

26. Aro AR, Smith J, Dekker J. Contextual evidence in clinical medicine and health promotion. Eur J Public Health. 2008;18(6):548-549.

27. Fisher EB, Boothroyd RI, Coufal MM, et al. Peer support for selfmanagement of diabetes improved outcomes in international settings. Health Aff (Millwood). 2012;31(1):130-139.

28. Standards of medical care in diabetes-2015: summary of revisions. Diabetes Care. 2015;38(Suppl):S4.

29. Andreae SJ, Halanych JH, Cherrington A, Safford MM. Recruitment of a rural, southern, predominantly African-American population into a diabetes self-management trial. Contemp Clin Trials. 2012; 33(3):499-506.

30. Piette JD, Resnicow K, Choi H, Heisler M. A diabetes peer support intervention that improved glycemic control: mediators and moderators of intervention effectiveness. Chronic Illn. 2013;9(4):258-267.

31. Moskowitz D, Thom DH, Hessler D, Ghorob A, Bodenheimer T. Peer coaching to improve diabetes self-management: which patients benefit most? J Gen Intern Med. 2013;28(7):938-942.

32. Ayala GX, Ibarra L, Cherrington AL, et al. Puentes hacia una major vida (bridges to a better life): outcome of a diabetes control peer support intervention. Ann Fam Med. 2013;13(Suppl 1)S9-S17.

33. Safford MM, Andreae S, Cherrington AL, et al. Peer coaches to improve diabetes outcomes in rural Alabama: a cluster randomized trial. Ann Fam Med. 2013;13(Suppl 1)S18-S26.

34. Tang TS, Funnell MM, Sinco B, Spencer MS, Heisler M. Peer-Led, Empowerment-Based Approach to Self-Management Efforts in Diabetes (PLEASED): a randomized controlled trial in an African American Community. Ann Fam Med. 2013;13(Suppl 1)S27-S35.

35. Zhong X, Wang Z, Fisher EB, Tanasugarn C. Peer support for diabetes management in primary care and community settings in Anhui Province, China. Ann Fam Med. 2013;13(Suppl 1)S50-S58.

36. Knox L, Huff J, Graham D, et al. What peer mentoring adds to already good patient care: implementing the Carpeta Roja peer mentoring program in a well-resourced health care system. Ann Fam Med. 2013;13(Suppl 1)S59-S65.

37. Goldman ML, Ghorob A, Hessler D, Yamamoto R, Thom DH, Bodenheimer T. Are low-income peer health coaches able to master and utilize evidence-based health coaching? Ann Fam Med. 2013;13(Suppl 1)S36-S41.

38. Yin J, Wong R, Au S, et al. Effects of providing peer support on diabetes management in people with type 2 diabetes. Ann Fam Med. 2013;13(Suppl 1)S42-S49.

39. Daaleman TP, Fisher EB. Enriching patient-centered medical homes through peer support. Ann Fam Med. 2013;13(Suppl 1)S73-S78.
40. Richman JS, Andreae S, Safford MM. Challenges of prolonged follow-up and temporal imbalance in pragmatic trials: analysis of the ENCOURAGE Trial. Ann Fam Med. 2013;13(Suppl 1)S66-S72.

41. Simmons D, Bunn C, Nakwagala F, et al. Challenges in the ethical review of peer support interventions. Ann Fam Med. 2013;13(Suppl 1):S79-S86.

42. Tang TS, Nwankwo R, Whiten Y, Oney C. Outcomes of a churchbased diabetes prevention program delivered by peers: a feasibility study. Diabetes Educ. 2014;40(2):223-230.

43. Gillon R. Medical ethics: four principles plus attention to scope. BMJ. 1994;309(6948):184-188.

44. Fisher EB, Ballesteros J, Bhushan N, et al. Beyond Efficacy: Key Features of Peer Support in Chronic Disease Prevention and Management. Health Aff (Millwood). In press.

45. Rosenthal EL, Wiggins N, Brownstein JN, et al. A Summary of the National Community Health Advisor Study: Weaving the Future. Tucson: Mel and Enid Zuckerman College of Public Health, University of Arizona, 1998.

46. Reinschmidt KM, Hunter JB, Fernández ML, Lacy-Martínez CR, Guernsey de Zapien J, Meister J. Understanding the success of promotoras in increasing chronic diseases screening. J Health Care Poor Underserved. 2006;17(2):256-264.

47. Cummings DM, Lutes LD, Littlewood K, Dinatale E, Hambidge B, Schulman K. EMPOWER: a randomized trial using community health workers to deliver a lifestyle intervention program in African American women with Type 2 diabetes: design, rationale, and baseline characteristics. Cont Clin Trials. 2013;36(1):147-153.

48. Kowitt SD, Urlaub D, Guzman-Corrales L, et al. Emotional support for diabetes management: an international cross-cultural study. Diabetes Educ. 2015;41(3):291-300.

49. Kim HS, Sherman DK, Taylor SE. Culture and social support. Am Psychol. 2008;63(6):518-526.

50. Ko GT, So WY, Tong PC, et al. From design to implementation-the Joint Asia Diabetes Evaluation (JADE) program: a descriptive report of an electronic web-based diabetes management program. BMC Med Inform Decis Mak. 2010;10:26.

51. Fisher EB, Chan J, Heisler M, Oldenburg B, Sartorius N. Peer Support As Intervention Strategy For Coexisting Depression And Chronic Disease: Illustrations From Diabetes. Symposium presented at the International Society for Affective Disorders. Berlin2014.

52. Peers for Progress. Global Evidence for Peer Support: Humanizing Health Care. Report from an International Conference. Leawood, Kansas: Peers for Progress, American Academy of Family Physicians Foundation; 2014.

53. Peers for Progress. What is Peer Support [video]? August 2015. http://peersforprogress.org/pfp-social-medial. Accessed August $17,2015$.

54. Peers for Progress, Peer Support: A Day in the Life: Promotores de Salud \& Community Health Workers [video]. February 2015 .http:/l peersforprogress.org/pfp-social-medial. Accessed Aug 17, 2015.

53. Rosenthal EL, Brownstein JN, Rush CH, et al. Community health workers: part of the solution. Health Aff (Millwood). 2010;29(7):1338-1342. 